\title{
ASMA CRÍTICA ASOCIADA A ASPERGILOSIS BRONCOPULMONAR ALÉRGICA: REPORTE DE UN CASO
}

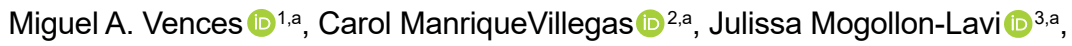 \\ Tatiana Arias-Rojas $\mathbb{1}^{2, a}$, Fernando Barriga Cari ${ }^{4, b}$ \\ 1 Departamento de Neurología, Hospital Nacional Edgardo Rebagliati Martins, Lima, Perú. \\ 2 Servicio de Neumología. Departamento de Neumología, Hospital Nacional Edgardo Rebagliati Martins, Lima, Perú. \\ 3 Departamento de Medicina Interna, Hospital Nacional Edgardo Rebagliati Martins, Lima, Perú. \\ ${ }^{4}$ Departamento de Cuidados Intensivos, Hospital Nacional Edgardo Rebagliati Martins, Lima, Perú. \\ a Médico/a residente; ${ }^{b}$ Médico asistente
}

\section{RESUMEN}

El síndrome de asma crítica es la peor consecuencia de una exacerbación aguda de asma. La aspergilosis broncopulmonar alérgica es una de las patologías más frecuentes que se mimetizan con este síndrome y está asociada a un alto riesgo de mortalidad cuando no se realiza el diagnóstico oportuno en pacientes asmáticos de difícil control. Presentamos el caso de un varón de 15 años que ingresó a la unidad de cuidados intensivos con clínica de asma crítica, donde se hizo un diagnóstico de aspergilosis broncopulmonar alérgica y respondió favorablemente con voriconazol y corticoides. En nuestro medio se debe considerar el diagnóstico de aspergilosis broncopulmonar alérgica en todo paciente con clínica de asma crítica o con una enfermedad pulmonar crónica de difícil control; el diagnóstico y el tratamiento oportunos mejoran la calidad de vida y el pronóstico de los pacientes.

Palabras clave: Asma; Aspergilosis Broncopulmonar Alérgica; Aspergillus fumigatus (fuente: DeCS BIREME).

\section{CRITICAL ASTHMA ASSOCIATED WITH ALLERGIC BRONCHOPULMONARY ASPERGILLOSIS: CASE REPORT}

\begin{abstract}
Critical asthma syndrome is the most severe consequence of an acute asthma exacerbation. Allergic bronchopulmonary aspergillosis is one of the most frequent pathologies that mimic critical asthma syndrome and is associated with a high mortality risk when timely diagnosis is not achieved in difficult-to-control asthmatic patients. We present the case of a 15 -year-old male who was admitted to the intensive care unit with critical asthma signs and symptoms, where a diagnosis of allergic bronchopulmonary aspergillosis was made. He responded favorably with voriconazole and corticoids. In our context, the diagnosis of allergic bronchopulmonary aspergillosis should be considered in all patients with critical asthma or with a chronic lung disease that is difficult to control. Early diagnosis and treatment improve the quality of life and prognosis of patients.
\end{abstract}

Keywords: Asthma; Allergic bronchopulmonary aspergillosis; Aspergillus fumigatus (source: MeSH NLM).

Citar como: Vences MA, Manrique-Villegas C, MogollonLavi J, Arias-Rojas T, Barriga Cari F. Asma crítica asociada a aspergilosis broncopulmonar alérgica: reporte de un caso. Rev Peru Med Exp Salud Publica. 2020;37(2):367-70. doi: https://doi.org/10.17843/ rpmesp.2020.372.4773

Correspondencia: Miguel Angel Vences; vens1793@hotmail.com

Recibido:30/08/2019 Aprobado: 06/05/2020 En línea: 12/06/2020

\section{INTRODUCCIÓN}

El síndrome de asma crítica está definido por una afección respiratoria grave y repentina que necesita un tratamiento agresivo y urgente ${ }^{(1)}$, por lo que la mayoría de casos requieren ingreso en la unidad de cuidados intensivos siendo considerada la peor consecuencia de una exacerbación aguda de asma ${ }^{(2)}$. Diversas enfermedades se pueden mimetizar clínicamente con el síndrome de asma crítica, siendo la aspergilosis broncopulmonar alérgica (ABPA) una de las más frecuentes y asociada a un alto riesgo de mortalidad cuando no se realiza el diagnóstico oportuno en pacientes asmáticos ${ }^{(1,3)}$.

Presentamos el caso de un varón con asma alérgica que cursó con clínica de asma crítica asociada a aspergilosis broncopulmonar alérgica y que respondió favorablemente al tratamiento antifúngico con voriconazol y corticoides. 


\section{REPORTE DE CASO}

Paciente masculino de 15 años, natural de Lima, con historia familiar de ambos padres con dermatitis atópica, contacto domiciliario con aves de corral y antecedente personal de rinitis, dermatitis atópica y asma alérgica diagnosticada cuando tuvo 3 años de edad, tratamiento habitual con fluticasona-salmeterol de $250 \mu \mathrm{g} 50 \mu \mathrm{g}$ /dosis (2 inhalaciones cada 12 horas) y $5 \mathrm{mg}$ diarios de prednisona, con adherencia al tratamiento.

En los últimos tres años presenta hospitalizaciones frecuentes de corta estancia, por aumento de episodios de broncoespasmo asociados a atelectasias, a pesar del tratamiento regular. Es evaluado por Inmunología clínica - Alergología, quienes realizan estudio con resultados de IgE sérica total de $1470 \mathrm{UI} / \mathrm{mL}$ y anticuerpos IgE específicos contra el alérgeno de Aspergillus fumigatus con un valor de 0,784 KUI/L, clasificado en el rango moderado bajo (clase 2). El servicio de neumología realizó estudios de tomografía torácita, test de caminata y espirometría, todos dentro de parámetros normales. Se clasifica el caso como asma persistente moderada, agregando Montelukast $10 \mathrm{mg}$ diarios a la terapia de mantenimiento.

En julio de 2019, el paciente ingresa a un hospital de la seguridad social de Lima referido de un centro de salud, por presentar desde hace un día, tos, disnea progresiva y un hallazgo radiográfico de neumomediastino y atelectasia de lóbulo inferior derecho. Al ingreso presentó polipnea, uso de musculatura accesoria, acidosis metabólica e hipoxemia severa sin respuesta satisfactoria a terapia inhalatoria intensa con beta 2 agonistas de acción corta y corticoides endovenosos (hidrocortisona $100 \mathrm{mg} / 8$ horas), con el diagnóstico inicial de asma crítica se decide el inicio de ventilación mecánica invasiva y sedación en la unidad de cuidados intensivos.

En la unidad de cuidados intensivos al examen físico se evidencia sibilantes espiratorios y, al evaluar la mecánica respiratoria del paciente, se evidencia compliance estática pulmonar normal, resistencia de la vía aérea incrementada y autopresión positiva al final de la espiración (PEEP) con hallazgo en tomografía de atelectasias en ambos lóbulos inferiores pulmonares. Se decide ventilación mecánica en modo volumen control con tidal $6 \mathrm{ml} / \mathrm{kg}$, frecuencia respiratoria: 12, I/E: 1:3, y PEEP: $10 \mathrm{cmH}_{2} \mathrm{O}$; se mantiene corticoterapia endovenosa (metilprednisolona $30 \mathrm{mg} / 12 \mathrm{~h}$ ), beta 2 agonistas de acción corta y se agrega bromuro de ipratropio.

Cursa con evolución tórpida, con persistencia de hipoxemia severa y presencia de neumotórax izquierdo (Figura 1), por lo que se coloca tubo de drenaje torácico y se realiza broncofibroscopia flexible con el objetivo de resolver atelectasias. Durante el procedimiento se evidencia broncorrea purulenta abundante que se logra aspirar parcialmente, con mejora parcial de la hipoxia y una tomografía con resolución parcial de la

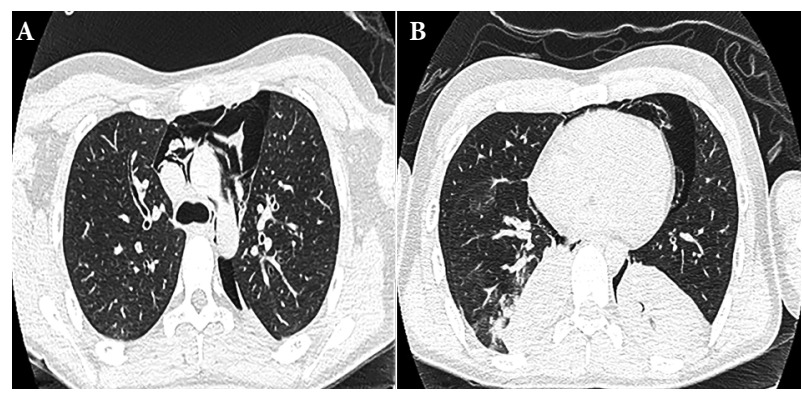

Figura 1. Tomografía torácica. A) Neumotórax moderado izquierdo, además de enfisema subcutáneo y neumomediastino. B) Parcial colapso pulmonar además de compromiso consolidativo con broncograma en las regiones posterobasales y atelectasias segmentarias lobares inferiores bilateral.

atelectasia, neumotórax residual laminar y neumomediastino de similares características. Tres días después el paciente cursa de manera estacionaria por lo que se decide suspensión de sedación y fisioterapia respiratoria.

Por antecedente referido y por una alta sospecha de aspergilosis broncopulmonar alérgica que condicionaría el aumento de los episodios de broncoespasmo, se le realiza un nuevo panel de estudio por inmunología clínica y alergología con los siguientes resultados: IgE sérica total de $1441 \mathrm{UI} / \mathrm{mL}$, anticuerpos IgE específicos contra el alérgeno de Aspergillus fumigatus con un valor de 58,8 KUI/L clasificado en rango muy alto (clase 5) y prueba intradérmica de escarificación para alergias (prick test) para Aspergillus que fue positiva con habón de $5 \mathrm{~mm}$.

En el examen microbiológico de las muestras tomadas en la broncofibroscopía, demostró al examen directo la presencia de filamentos hialinos septados y cabezas aspergilares, creciendo en la resiembra colonias planas de aspecto aterciopelado de color verde con halo claro características de Aspergillus fumigatus (Figura 2) por lo que se estableció el diagnóstico de ABPA seropositiva aguda.

Al quinto día de ingreso a cuidados intensivos, el paciente presentó fiebre, hipoxemia, aumento de gradiente alveolo capilar, aumento de neumotórax, neumomediastino y enfisema subcutáneo sin aparente obstrucción de tubo de drenaje torácico. Por sospecha de neumonía asociada a ventilador mecánico como complicación, se inició cobertura antibiótica empírica de amplio espectro y por el diagnóstico de ABPA se inició antifúngico sistémico con voriconazol 200 mg/ 12 horas debido a no disponibilidad de itraconazol; completando esquema antibiótico por 7 días con mejoría clínica y radiológica (Figura 3) por lo que se decide destete, extubación y retiro de tubo de drenaje torácico.

$\mathrm{Al}$ alta de la unidad de cuidados intensivos, se decidió mantener con prednisona vía oral a $1 \mathrm{mg} / \mathrm{kg}$ de peso con disminución gradual de dosis; además de mantener el voriconazol $200 \mathrm{mg} / 12 \mathrm{~h}$ por 16 semanas debido a que tenía un 


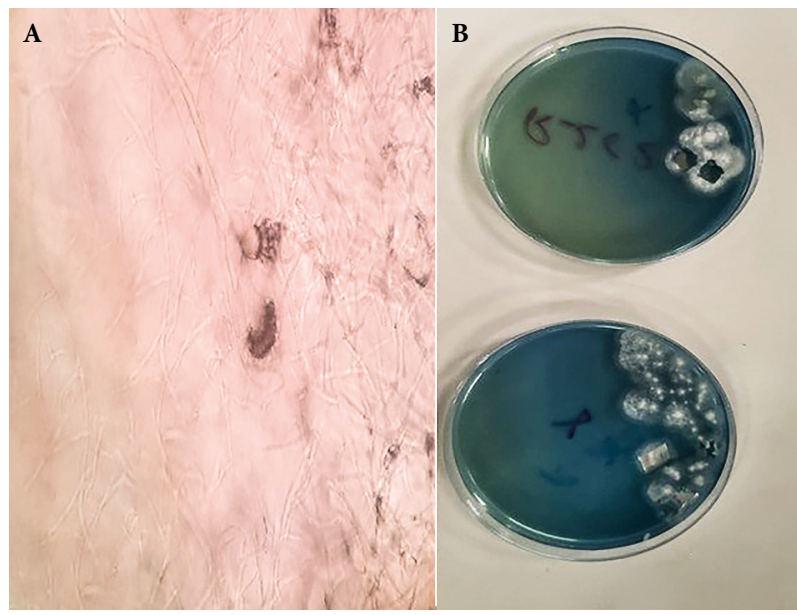

Figura 2. Examen microbiológico. A) Examen directo aclarado con $\mathrm{KOH}$ se observan filamentos hialinos septados y cabezas aspergilares. B) Resiembra con presencia de colonias planas de Aspergillus fumigatus, de aspecto aterciopelado de color verde con halo claro.

buen patrón de sensibilidad de acuerdo al fungigrama (CIM 0,12). Al mes del alta, el paciente presentó mejoría clínica notable con ausencia de episodios de broncoespasmo y con un descenso de $\operatorname{IgE}$ (550 UI/mL).

\section{DISCUSIÓN}

Aspergillus fumigatus es la especie más frecuente del género Aspergillus, es un hongo saprófito que se adquiere por inhalación y se encuentra en mayores concentraciones en

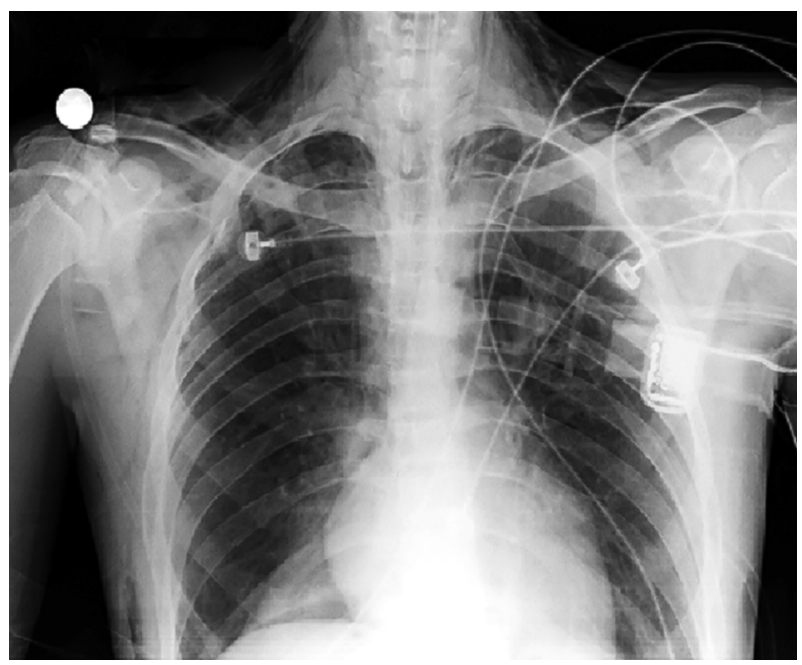

Figura 3. Radiografía torácica. Imagen de neumotórax izquierdo resuelto con pulmón izquierdo expandido en presencia de tubo de drenaje torácico de proyección habitual y además se observa atelectasias segmentarias lobares inferiores que persisten, pero de forma mínima residual. ambientes con alto grado de humedad y polvo, sin ser patológico en la mayoría de ocasiones ${ }^{(4)}$. El compromiso respiratorio ocasionado por la aspergilosis incluye un amplio espectro de manifestaciones clínicas, siendo la aspergilosis broncopulmonar alérgica la manifestación más compleja de estas entidades.

Se desconoce la prevalencia mundial de ABPA. Existen reportes epidemiológicos en los que consideran que Aspergillus fumigatus coloniza el árbol respiratorio del 25\% de asmáticos; sin embargo, solo $2 \%$ de los pacientes llegan a desarrollar ABPA. Actualmente se ha estimado que la carga global de ABPA supera los 4,8 millones en el mundo, siendo una entidad rutinariamente subdiagnosticada que afecta la calidad de vida de los pacientes ${ }^{(5)}$.

La ABPA se caracteriza por el compromiso pulmonar resultante de hipersensibilidad a las esporas fúngicas que generalmente se eliminan del pulmón a través de mecanismos innatos y mecánicos, pero cuando se encuentran en grandes cantidades la respuesta innata puede no ser suficiente para eliminar las esporas y la sensibilización alérgica se hace cargo. La interacción de estos antígenos con los linfocitos genera una respuesta inmune caracterizada por la producción de interleucinas, las células Th2 son atraídas a las vías respiratorias incrementando tanto la síntesis de IgE por las células B como la atracción de eosinófilos al tejido, alterando la depuración mucociliar que ocasiona impactación mucosa con obstrucción de la vía aérea generando finalmente remodelación de las vías respiratorias y perpetuando así el daño de la vía aérea ${ }^{(6)}$.

La manifestación clínica de la ABPA es muy variada, desde una enfermedad leve hasta casos mortales y depende de la respuesta inmune y de las comorbilidades, siendo el asma y la fibrosis quística las entidades más frecuentes; sin embargo, existen reportes de casos de esta afección sin ningún antecedente de enfermedad respiratoria.

Los exámenes laboratoriales en la ABPA tienen una sensibilidad y especificidad muy bajas individualmente, por lo que los criterios diagnósticos de ABPA se han ido modificando desde los primeros reportes descritos en 1952 por Hinson y en 1969 por Slavin, hasta el 2013 cuando la Sociedad Internacional de Micología Humana y Animal (ISHAM Working Group) establece los criterios diagnósticos vigentes de la $\mathrm{ABPA}^{(3)}$. Se clasifican en tres criterios: 1) condiciones predisponentes: asma y fibrosis quística; 2) criterios obligatorios: una prueba cutánea de Aspergillus tipo I positiva o niveles elevados de IgE contra Aspergillus fumigatus, y como segundo criterio la presencia de niveles elevados de IgE total de más de 1000 UI / ml (a menos que todos los otros criterios se cumplen, los niveles totales de IgE pueden ser inferiores a $1000 \mathrm{UI} / \mathrm{ml}$ ); 3) otra condición, que deben ser al menos dos de las siguientes: presencia de anticuerpos IgG o anticuerpos precipitantes contra Aspergillus fumigatus, presencia de opacidades pulmonares fugaces o fijas en la radiografía de tórax 
consistentes con ABPA y recuento de eosinófilos de más de 500 células/ $\mu \mathrm{L}$ en pacientes sin tratamiento previo con esteroides (puede ser un valor histórico).

La estadificación convencional de ABPA, incluye cinco estadios caracterizados por el correlato temporal de las manifestaciones clínicas, radiológicas y serológicas ${ }^{(3,7)}: 1$ ) agudo: se diagnostica ABPA en el paciente y se observan todas las características típicas; 2) remisión: asintomático con asma controlada sin nuevos hallazgos radiológicos y sin aumento de la IgE total durante un mínimo de seis meses; 3) exacerbación: aparecen nuevos infiltrados pulmonares con eosinofilia y se duplica la IgE en el nivel de remisión; 4) asma dependiente de corticoide: los pacientes se vuelven dependientes del tratamiento con corticoides; 5) estadio final: hallazgos de fibrosis o cavitación crónica y los parámetros serológicos suelen ser negativos.

En este reporte se observa que el paciente era asmático, como criterio predisponente, y presentó ambos criterios obligatorios de diagnóstico, cumpliendo además criterios para el estadio agudo. El diagnóstico final fue de aspergilosis broncopulmonar alérgica seropositiva aguda. Se asume que fue colonizado por Aspergillus mucho tiempo antes y que en los últimos tres años ha desarrollado la ABPA, generando el aumento de los broncoespasmos y haciendo que el asma clasifique por su severidad como persistente moderada.

Los glucocorticoides sistémicos son los fármacos más eficaces para el tratamiento de ABPA a una dosis inicial de $0,5 \mathrm{mg} / \mathrm{kg}$ al día durante 14 días, con reducción gradual y suspensión en tres meses ${ }^{(7)}$. La terapia antifúngica con azo-

\section{REFERENCIAS BIBLIOGRÁFICAS}

1. Kenyon N, Zeki AA, Albertson TE, Louie S. Definition of critical asthma syndromes. Clin Rev Allergy Immunol. 2014;48(1):1-6. doi: 10.1007/ s12016-013-8395-6.

2. Schivo M, Phan C, Louie S, Harper RW. Critical asthma syndrome in the ICU. Clin Rev Allergy Immunol. 2015; 48(1):31-44. doi: 10.1007/ s12016-013-8394-7.

3. Agarwal R, Chakrabarti A, Shah A, Gupta D, Meis JF, Guleria R, et al. Allergic Bronchopulmonary Aspergillosis: Review of Literature and Proposal of New Diagnostic and Classification Criteria. Clin Exp Allergy. 2013; 43(8):850-873. doi: 10.1111/cea.12141.

4. Knutsen AP. Allergic bronchopulmonary aspergillosis. Clin Exp Allergy. 2015; 45(2):298-9. doi: 10.1111/cea.12459.

5. Stevens DA, Kan VL, Judson MA, Morrison VA, Dummer S, Denning DW, et al. Practice guidelines for diseases caused by Aspergillus. Clin Infect Dis. 2000; 30(4):696-709. doi: 10.1086/313756.

6. Roy RM, Klein BS. Fungal glycan interactions with epithelial cells in allergic airway disease. Curr Opin Microbiol. 2013;16(4):404-8. doi: 10.1016/j. mib.2013.03.004. les incluidos el Itraconazol y Voriconazol, que actúan mediante la reducción de la carga fúngica ayudando a controlar el estímulo antigénico y por lo tanto disminuir la respuesta inflamatoria; se utilizan en el tratamiento asociados a corticoides para disminuir el riesgo de exacerbación de ABPA ${ }^{(8)}$.

Se ha observado en el seguimiento ambulatorio de los pacientes: una buena respuesta clínica y radiológica, una disminución de los niveles séricos de IgE y un retorno a los niveles normales de eosinófilos en sangre. Estas características son considerados tradicionalmente como factores de remisión de ABPA, sin embargo, a la fecha, los resultados son no concluyentes ${ }^{(9,10)}$. Agarwal y col, realizaron un estudio donde fijó como punto de corte la disminución del 35\% de IgE sérica a las seis semanas como criterio de remisión, observando que no todos los pacientes con mejoría clínica y radiológica cumplían con este criterio, siendo más sensible cuando el valor basal de IgE era mayor a $2500 \mathrm{UI} / \mathrm{ml}^{(11)}$.

En el presente caso se consideró continuar con el uso de voriconazol, debido a su buen patrón de sensibilidad, buena tolerancia gastrointestinal y biodisponibilidad ${ }^{(12)}$, con una reducción de más del 50\% de IgE sérica y ausencia de episodios de broncoespasmo al primer mes del seguimiento ambulatorio.

En conclusión, reportamos un caso de ABPA seropositivo agudo con una presentación clínica de asma crítica potencialmente mortal. Es importante considerar como posibilidad diagnóstica a la ABPA, en todo paciente con enfermedad pulmonar crónica de difícil control. El diagnóstico y tratamiento oportuno mejora la calidad de vida y el pronóstico de estos pacientes.

7. Patel A R, Patel A R, Singh S, KhawajaI. Treating Allergic Bronchopulmonary Aspergillosis: A Review. Cureus 2019; 11(4): e4538. doi: 10.7759/cureus.4538.

8. Stevens DA, Schwartz HJ, Lee JY, Moskovitz BK, Jerome DC, Catanzaro A, et al. A randomized trial of itraconazole in allergic bronchopulmonary aspergillosis. N Engl J Med. 2000;342(11):756-62. doi: 10.1056/ NEJM200003163421102.

9. Ricketti AJ, Greenberger PA, Patterson R. Serum IgE as an important aid in management of allergic bronchopulmonary aspergillosis. J Allergy Clin Immunol. 1984;74(1):68-71. doi: 10.1016/0091-6749(84)90089-7.

10. Agarwal R. Allergic bronchopulmonary aspergillosis. Chest. 2009;135(3):805-826. doi: 10.1378/chest.08-2586.

11. Agarwal R, Gupta D, Aggarwal AN, Saxena AK, Saikia B, Chakrabarti A, Jindal SK. Clinical significance of decline in serum IgE levels in allergic bronchopulmonary aspergillosis. Respir Med. 2010, 104(2):204-10. doi: 10.1016/j.rmed.2009.09.005.

12. Clancy CJ, Nguyen MH. Long-term voriconazole and skin cancer: is there cause for concern? Curr Infect Dis Rep. 2011;13(6):536-43. doi: 10.1007/s11908-011-0220-x. 